\title{
Remarques sur le breton parlé à Plaudren
}

Remarks on the Breton spoken in Plaudren

\section{Anne-Marie Quéré}

\section{CpenEdition}

\section{Journals}

Édition électronique

URL : https://journals.openedition.org/lbl/2155

DOI $: 10.4000 / \mathrm{lbl} 2155$

ISSN : 2727-9383

\section{Éditeur}

Université de Bretagne Occidentale - UBO

\section{Édition imprimée}

Date de publication : 1 novembre 2011

Pagination : 111-122

ISBN : 978-2-901737-92-6

ISSN : $1270-2412$

\section{Référence électronique}

Anne-Marie Quéré, «Remarques sur le breton parlé à Plaudren », La Bretagne Linguistique [En ligne], 16। 2011, mis en ligne le 01 mai 2021, consulté le 21 mai 2021. URL : http://journals.openedition.org/lbl/ 2155 ; DOl : https://doi.org/10.4000//bl.2155

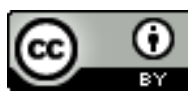

La Bretagne Linguistique est mise à disposition selon les termes de la Licence Creative Commons Attribution 4.0 International. 
Anne-Marie QUÉRÉ*

\title{
Remarques sur le breton parlé à Plaudren
}

\begin{abstract}
P au nord de Vannes, au bord des Landes de Lanvaux, à la frontière de la zone bretonnante. Les communes limitrophes, Plumelec au nord et Trédion à l'est, sont en pays gallo.

Le breton de Plaudren est celui de ma famille paternelle, celui que j'entendais dans mon enfance, chez mes grands-parents, chez mes oncles et tantes cultivateurs. Moi-même, je n'ai pas été élevée en breton, mais j'ai aimé répéter des phrases, des proverbes, des chansons, noter les tournures que nous entendions dans la bouche de nos parents. Ce n'est pas «ce breton-là» que j'ai appris dans les stages et autres cours du soir, mais c'est celui que j'avais dans la tête et dans le cœur, si différent du «breton du Finistère ». J'ai réalisé mon premier enregistrement en 1979, auprès de mon père (né en 1912). Bien plus tard, alors que je suivais les cours de dialectologie de Jean Le Dû, à Brest, j'ai voulu naturellement travailler sur le vannetais et j'ai eu l'occasion d'enregistrer certains des derniers bretonnants naturels de la commune de Plaudren, essentiellement des gens de ma famille, ce qui a beaucoup facilité les choses.
\end{abstract}

Aujourd'hui, la pratique du breton y est très faible. La transmission s'est arrêtée après la Seconde Guerre mondiale : ainsi mes

\footnotetext{
* Professeur d'histoire-géographie et breton en lycée
} 
cousins, ceux de mon âge, qui ont toujours vécu à Plaudren, ne connaissent pas le breton. La disparition progressive des anciens, l'arrivée de nouvelles familles venues s'installer dans les nouveaux lotissements tout en travaillant à Vannes font que l'on n'entend plus parler breton spontanément à Plaudren.

Le corpus dont je dispose est le résultat d'enregistrements réalisés auprès de plusieurs personnes de ma famille qui, pour la plupart, sont nées et ont toujours vécu à Plaudren. Ce corpus comprend deux types d'entretien :

- des entretiens en continu, des "histoires de vie" auprès d'informateurs nés avant la Première Guerre mondiale ;

- des phrases à traduire auprès d'informateurs plus jeunes, nés dans les années 1920 et 1930 :

soit dans le cadre du projet du Nouvel Atlas Linguistique de Basse-Bretagne,

soit, de manière plus informelle, en soumettant à mes informateurs des phrases variées, relevées parfois dans la grammaire de Francis Favereau, ou encore des phrases du Breton usuel indispensable aux touristes, commerçants, hommes d'affaires de Loeiz Herrieu (1934).

Je poursuivais ces enquêtes uniquement pour le plaisir, sans intention de communiquer ou de formuler quelque conclusion que ce soit, lorsque Jean Le Dû m'a invitée à présenter ce parler de Plaudren. J'ai donc choisi de confronter ce que j'ai pu observer aux différentes grammaires, particulièrement celle de Francis Favereau, Grammaire du breton contemporain (1997) et celle de A. Guillevic et P. Le Goff, Grammaire bretonne du dialecte de Vannes (1902), mais aussi aux articles de Jean-Pierre Ledunois «Grammaire et dialectologie» (Bretagne Linguistique 13) et de Jean-Yves Plourin «La phrase bretonne comprenant le verbe être au présent de l'indicatif» (Bretagne linguistique 11); ces "Remarques sur le breton parlé à Plaudren» sont donc des remarques grammaticales qui porteront sur les points suivants :

- les conjugaisons et leur importance respective dans le parler de Plaudren ;

- les formes du verbe être : l'utilisation de ema et vez. 
Je précise, que, pour la transcription des exemples, j'utilise l'orthographe fixée par Guillevic et Le Goff dans leur grammaire (opus cité).

\section{Les conjugaisons et leur importance respective dans le parler de Plaudren}

On sait qu'il existe trois façons de conjuguer un verbe en breton : mon propos est d'envisager leur importance respective dans le parler de Plaudren, et par conséquent la place du verbe dans les indépendantes et les principales. Pour cela, j'ai choisi de confronter mes observations aux règles énoncées par les grammairiens.

\section{Ce que disent les grammairiens}

Pour Guillevic et Le Goff (opus cité p. 38), il y a en breton deux conjugaisons des verbes : la conjugaison personnelle, où la forme du verbe varie suivant le nombre et la personne du sujet, et la conjugaison impersonnelle, où le verben'a qu' une seule forme à chaque temps, celle de la troisième personne du singulier... La conjugaison personnelle s'emploie après la négation et les conjonctions de subordination, après le complément direct, l'attribut, et ordinairement après l'adverbe, le complément indirect et les propositions subordonnées.

Je n'ai pas relevé dans la grammaire en question d'indication sur l'importance respective de ces deux conjugaisons. Néanmoins, dans un chapitre intitulé «Syntaxe du verbe» (p. 88) les auteurs notent que «après un adverbe, un complément indirect ou circonstanciel, une courte proposition subordonnée... on peut continuer par le sujet» et donc utiliser la conjugaison impersonnelle.

Exemples :

Liès me ouil / Souvent je pleure

De Zoé me gan melodi / Je chante les louanges de Dieu Mar det, hui e vou lahet / Si vous y allez, vous serez tué

Quant à la conjugaison que 1'on appelle "périphrastique», les auteurs l'évoquent rapidement dans le cadre du paragraphe intitulé «conjugaison impersonnelle»: «on emploie aux temps simples le verbe gobér (faire) précédé de l'infinitif: kañnein e hran (je chante)». 
Pour Francis Favereau, la conjugaison périphrastique, c'està-dire la structure à infinitif en tête «est assez largement usitée... puisqu' on l'emploie dans quelque $6 \%$ du total des indépendantes ou des principales ici considérées (Poher).»

La conjugaison impersonnelle s'emploie lorsque le sujet est exprimé et mis en relief suivant l'ordre SVO (p. 190). Cette structure qui met le sujet en tête se retrouve dans $25 \%$ des cas (p. 296). Un peu plus loin (p. 298), il note que cet emploi du sujet en tête, notamment d'un pronom personnel sujet, "varie beaucoup en fonction des parlers, voire des générations : il reste plus emphatique, car plus rare, en KLT qu'en vannetais (où l'on admet bien plus souvent le sujet en tête, sous l'influence du français voisin, dit-on)».

La conjugaison personnelle s'emploie «lorsque le sujet n'est exprimé que par la marque de personne, après la négation, après un COD ou un COI, l'attribut ou l'adverbe; cette conjugaison se retrouve donc largement majoritaire dans le corpus du Poher».

On retrouve cette conjugaison dans le cas des structures dépendantes ou indépendantes après ha ou hag (p. 290).

Pour Jean-Pierre Ledunois, la conjugaison est dite nominale « lorsque la personne apparaît en tête du syntagme verbal», strictement verbale «lorsque la personne apparaît à l'intérieur du verbe sous forme de désinence», périphrastique «lorsque la personne apparaît à l'intérieur d'un auxiliaire».

Il a répertorié tous les verbes de l'enregistrement des Histoires en breton vannetais. Dans ce corpus, la conjugaison nominale est largement majoritaire ( $60 \%$ des cas), beaucoup plus fréquente que dans le breton du Poher: "la conjugaison nominale domine en vannetais».

\section{Et à Plaudren}

- La conjugaison périphrastique :

Je n'ai relevé que deux fois la conjugaison dite périphrastique : une fois dans le corpus "en continu" : tostad e ra en noz / la nuit arrive; une fois dans le cadre des traductions soumises à mes informateurs : berr e en deieù, komansein e ra hiraat / les jours sont courts, mais ils commencent à rallonger. 
- La conjugaison impersonnelle :

La conjugaison impersonnelle (nominale selon J.-P. Ledunois) est la plus employée, que ce soit dans les récits en continu ou dans les phrases à traduire. La plupart du temps, donc, la personne apparaît en tête du syntagme verbal, le COD et le COI sont alors relégués après le verbe, comme en français.

Exemples dans les récits en continu : il s'agit de récits de vie, des souvenirs de l'ancien temps autour du travail, des fêtes, etc.

Germaine P., née en 1913 à Plaudren où elle tenait un café avec son mari, forgeron, évoque son enfance :

Ha ni e hoarié... Marcel ag André e hoarié genemb, ér prat... Hanéh e hré ér vouillen, haneh e hré pon-eu. Me Marcel fôte ket jamez kousi é zorneù ha mé laré d'hou : te vou beleg té. Ni o aval berdir...

(Et nous, on jouait... Marcel et André jouaient avec nous dans le pré... Il faisait des ponts dans le ruisseau. Mais Marcel ne voulait jamais se salir les mains et je lui disais : toi tu seras curé. On était comme des frères...)

Mes desket galleg get unan, ur voazinez o etalomb, er Hoh Prat; mes desket galleg geti; hag hé gonzé galleg dein, pe ouié ket breton...à la fin, mé zeské, mé zivizé avaldi; geti mé teské galleg...

(J'ai appris le français avec une voisine; et elle me parlait français puisqu'elle ne savait pas le breton... à la fin, j'apprenais, je parlais comme elle; c'est avec elle que j'apprenais le français.)

- Exemples de phrases soumises à la traduction :

Mes informateurs sont des personnes nées en 1920, 1926 et 1932, des gens de ma famille que je connais bien. Je les avais sollicités dans le cadre des enquêtes élaborées pour le Nouvel Atlas Linguistique de Basse-Bretagne et lorsque cette enquête a marqué une pause pour l'exploitation des données, j'ai décidé de poursuivre mes investigations. J'ai relevé des exemples dans la grammaire de Francis Favereau (opus cité), en les modifiant parfois pour les adapter au contexte. Ce que $\mathrm{j}$ 'ai obtenu est bien évidemment très éloigné du parler du Poher, et confirme les remarques que l'on peut faire sur l'utilisation de la conjugaison impersonnelle : 
En dud e ge pell ar droed gueharall (à Plaudren)

'Les gens allaient loin à pied autrefois'

Pell'h ae an dud war an troad (FF p. 291)

Diskiñ 'rae ar vugale gante (FF p. 302)

'Les enfants apprenaient grâce à eux (aux instituteurs)'

Ar vugale a zeske get er mestr skol (Plaudren)

Tapet ' $n$ 'eus hennezh ahanon (FF p. 291)

'Celui-là, il m'a bien eu'

Hane de me bet prop (Plaudren)

Gant ar saout 'veze laezh (FF p. 291 )

'Les vaches donnaient du lait autrefois'

Er seu e re leah guerahal

Ajoutons quelques exemples entendus :

En dud fatek fonab a bermen

'Les gens se fatiguent vite aujourd'hui'

Hi gemere soegn hag he broh velouz

'Elle prenait soin de sa robe de velours'

Te oui asset

'Tu le sais bien pourtant'

Ni e grolle guerahal

'Tu parles si on dansait autrefois'

Cette conjugaison impersonnelle est donc largement utilisée dans les principales et indépendantes, même lorsque le sujet n'est pas en tête de phrase, après un COI, un adverbe, ou une subordonnée. Ceci est conforme à ce que l'on peut lire dans la grammaire du vannetais (opus cité p. 88).

Après un COI ou un adverbe (les exemples qui suivent sont extraits des récits “en continu”) :

Mar e huéh er vaouez ieùank e ouilé

'Parfois, la jeune mariée pleurait' 
Geti me teské galleg

'C'est avec elle que j'apprenais le breton'

Er Hoh Prat, me gozé galleg, get Memé.

'Au Vieux Pré, je parlais français avec Mémé'

Hanter hant vléad zo bermen, me oé get ma zud en ur penhér, anauet «er Hoh Prat»

'Il y a maintenant cinquante ans, je vivais avec mes parents dans une ferme appelée "Le Vieux Pré»"

De bemzeg vlé, de houel Yann, gouléet on bet get ur mestr, e bro Kergonan, parrez Plaurén

'À 15 ans, j'ai été embauché par un paysan de Kergonan à Plaudren'

Er hué kentan, me laro hoah dis, ur pennad a mem buhé

'La prochaine fois, je te raconterai encore une partie de ma vie'

Arhoah, mem brereg a breno un oto

'Demain, mon beau-frère achètera une voiture'

J'ai relevé une fois la structure $a d v+v+s$ :

Arse e evo he hafé bemdé get un dapenn lagout a-barh

'Comme ça elle boira tous les jours son café avec la goutte dedans'

Après une subordonnée, dans le cas où la principale suit une subordonnée, je n'ai jamais relevé la structure $\mathrm{SUB}+\mathrm{e}+\mathrm{V}$; le sujet reste en tête de la principale.

Dans les réponses au questionnaire élaboré dans le cadre du Nouvel Atlas Linguistique de Basse Bretagne:

P'on klañ, me chom é ma gulé

'Quand je suis malade, je reste au lit'

Pa vein prest, me gei genis

'Quand je serai prêt, j'irai avec toi' 
Pa mes hoar, me ga de gerhed

'Quand j'ai le temps, je vais marcher'

Pa sonen, rah en dud e grolle

'Quand je chantais, tout le monde dansait'

Dans les récits "en continu":

Agost ma ous aman, te chomo da zèbein genem

'Puisque tu es là, tu vas rester manger avec nous'

Ha ma kargen ket mat, me zad e skoé un taul ar er pen de mem bah, mem freilh

'Et si je ne chargeais pas bien, mon père donnait un coup sur le bout de mon bâton, mon fléau'

J'ai relevé une seule fois dans un enregistrement réalisé en 1981, auprès de mon père (né en 1912) :

Ma karez bermen, éh an d'achiù aveid hiniù

'Si tu veux maintenant, je vais m'arrêter pour aujourd'hui'

- La conjugaison personnelle, "strictement verbale" :

Donc comme la plupart du temps, on utilise la forme impersonnelle avec sujet devant le verbe, je n'ai pratiquement jamais rencontré cette conjugaison personnelle dans le cadre de structures où le verbe suit un COD, un COI, un attribut ou un adverbe, à la forme affirmative bien sûr. Dans ce cas le verbe est en première position ou précédé de ha ou hag.

Le verbe est en première position, dans les exemples ci-dessous qui sont extraits des récits de vie de mes informateurs. Ceci est dû peut-être au fait qu'il s'agisse des verbes "forts" - bezañ, mont, dont, gallout, rankout - dont parle Francis Favereau (opus cité p. 318) :

É ta ar gouiañ

'L'hiver arrive'

E oé rah en dud er prad aveid er foen. E oé er mestr, un den iuel ha nerhus

'Il y avait beaucoup de monde dans le pré pour le foin. Il y avait le patron, un homme grand et fort' 
On oeit a nezé da Gerare de huéh vlé betag pearzek vlé 'Je suis donc allé à Kerarhaye de six à quatorze ans'

Après ha ou hag le verbe est à la conjugaison personnelle. Francis Favereau (opus cité p. 293) note que cette structure (avec verbe conjugué) est la plus courante, tant dans le corpus étudié du Poher ou en breton fañch, que dans le breton spontané (notamment des médias), ou même en breton écrit. C'est ce que j'ai observé lorsque j'ai proposé à mes informateurs des phrases commençant par et ou donc; mais uniquement si le sujet est un pronom personnel à la $3^{\mathrm{e}}$ personne du singulier ou du pluriel :

Ha gomanse troein en dro di

'Et il commençait à lui tourner autour'

Hag é zè geti ér gér

'Et il revenait avec elle à la maison'

Ha lennent passab

'Et ils lisaient beaucoup'.

Mais :

Ha me houlennou getou

'Et je le lui demanderai'

Donc, la conjugaison personnelle - à l'affirmatif, bien sûr - est peu employée et c'est la conjugaison impersonnelle qui domine largement ici à Plaudren, même si la phrase commence par un autre élément que le sujet. Le verbe n'est donc pas forcément en deuxième position, ce qui est conforme à la règle énoncée par Guillevic et Le Goff (opus cité p. 88). Mais selon Loeiz Herrieu, tout cela n'est pas très «celtique» et il note dans son Manuel de breton usuel (Éditions Dihunamb, 1934, Lorient) p. 20: «On doit éviter le plus possible d'employer la conjugaison impersonnelle qui enlève à la phrase son cachet breton» ou encore «la phrase bretonne ne peut se construire élégamment sur le modèle de la phrase française où le sujet précède le verbe»... 


\section{Le verbe être : l'emploi de emañ et bez}

\section{Emañ}

Jean-Yves Plourin dans son article «la phrase bretonne comprenant le verbe être au présent de l'indicatif» (opus cité) note, à propos de ema, que :

- «le vannetais, comme le Trégor et la Haute-Cornouaille n'use que des formes anciennes, les troisièmes personnes du singulier et du pluriel» (p. 284$)$

- «l'état temporaire est signalé... avec une fréquence supérieure à la moyenne dans le sud-est, par EMA» (p. 286) : ema prest / il est prêt.

Il relève aussi un exemple de la grammaire de Guillevic et Le Goff : Me gréd é ma klañ / Je crois qu'il est malade. Ces auteurs signalent que l'on peut dire aussi bien rak ma mant klañ que rak man dint klañ.

- «La situation dans l'espace est nécessairement un évènement provisoire» : Ema Yann ba'r gêr.

- «L'action ponctuelle envisagée comme inachevée s'exprime à l'aide de la tournure progressive» : Ema Yann o tont / Jean vient.

- Et à Plaudren :

Dans le corpus dont je dispose, la forme ema est très peu utilisée. À Plaudren, comme dans le reste du Vannetais, on n'use que des troisièmes personnes du singulier et du pluriel. Ainsi, on dit :

- Emen ous-te? On er gêr

- Où es tu ? Je suis à la maison

- Emen ema ? Ema er gêr

- Où est-il ? Il est à la maison.

- On é tèbein, ous é tèbein, ema é tèbein

- Je suis en train de manger, tu es en train de manger, il est en train de manger.

Pour signaler "l'état temporaire", j'ai relevé deux fois l'utilisation de ema :

- Hiniù ema klañ

- Aujourd'hui, il est malade 
- Kasimant sur ema vrai

- C'est probablement vrai et aussi :

- Mes me on okupet a mamm, gui m'en dé klañ

- Je me suis occupée de ma mère depuis qu'elle est malade.

Cela confirmerait ce que dit Jean-Yves Plourin : «Le locuteur a donc le choix, selon le contexte et l'opinion qu'il veut exprimer.»

- L'utilisation de la forme bez :

Cette forme est pratiquement absente de mon corpus, que ce soit pour exprimer la fréquence, l'habitude :

Me oé lies klañ

'J'étais souvent malade'

Pen dé tennet er guin, faote en ived

'Quand le vin est tiré, il faut le boire'

$P$ 'o en assemblée, ne renteein ket er gêr irok hueh er

'Quand il y avait une fête, je ne rentrais pas à la maison avant 6 heures'

ou pour exprimer l'indéfini "on" et donc l'idée de généralisation :

Guérahal, ni drohe er bléad get ur falz

'À cette époque-là, on coupait le blé à la faucille'

Arlerh ma nes dornet, ni zèbé ioh ensemble

'Après le battage, on mangeait tous ensemble'

Pa dén e sell a dreist er hé, den e uel asset e bark e zo sal

'Quand on regarde par-dessus la haie, on voit bien que son champ est sale'.

Cependant, j'ai rencontré cette forme fréquentative dans une chanson, qui m'a été chantée par une de mes informatrices :

Ha pe ver get er pred, nitra ne dalv ur lomig a sistr

'Et quand on se met à table, rien ne vaut un verre de cidre' ou encore dans un proverbe :

Pe vé laket gouil é leah, é dro a-benn

'Quand on met du ferment dans le lait, ça tourne, tout de suite'. 
Pour conclure, le parler de Plaudren se rattache bien aux parlers du Haut-Vannetais. Mais la situation géographique de la commune - à la frontière du pays gallo - permet peut-être d'expliquer l'influence du français sur la syntaxe. Ceci pourrait être approfondi en poursuivant l'enquête sur certains points de grammaire et sur le lexique et aussi en menant des comparaisons avec le parler des communes voisines du canton de Grandchamp. 